\title{
Phase behavior of symmetric linear multiblock copolymers
}

P. E. Theodorakis ${ }^{1,2,3}$ and N. G. Fytas ${ }^{4}$

1 Faculty of Physics, University of Vienna, Boltzmanngasse 5, A-1090 Vienna, Austria, EU

2 Institute for Theoretical Physics and Center for Computational Materials Science, Vienna University of Technology,

Hauptstraße 8-10, A-1040 Vienna, Austria, EU

-3. Vienna Computational Materials Laboratory, Sensengasse 8/12, A-1090 Vienna, Austria, EU

Department of Materials Science, University of Patras, 26504 Patras, Greece, EU

PACS 36.20.-r - Macromolecules and polymers

PACS 64.75.Yz - Self-assembly

PACS 64.75. Gh - Phase separation and segregation in model systems (hard spheres, LennardJones, etc.)

\begin{abstract}
Molecular dynamics simulations are used to study the phase behavior of a single linear multiblock copolymer with blocks of A- and B-type monomers under poor solvent conditions, varying the block length $N$, number of blocks $n$, and the solvent quality (by variation of the temperature $T$ ). The fraction $f$ of A-type monomers is kept constant and equal to 0.5 , and always the lengths of $\mathrm{A}$ and B blocks were equal $\left(N_{A}=N_{B}=N\right)$, as well as the number of blocks $\left(n_{A}=n_{B}=n\right)$. We identify the three following regimes where: (i) full microphase separation between blocks of different type occurs (all blocks of A-type monomers form a single cluster, while all blocks of B-type monomers form another), (ii) full microphase separation is observed with a certain probability, and (iii) full microphase separation can not take place. For very high number of blocks $n$ and very high $N$ (not accessible to our simulations) further investigation is needed.
\end{abstract}

The phase behavior of block copolymers has recently attracted much interest in experimental and theoretical studies [1 46]. For melts of multiblock copolymer chains the phase diagram almost resembles the phase diagram of that with diblock copolymer chains; The lamellar structure is expected for the most symmetrical case, an approach which is particularly valid in the strong segregation limit 33 . In the case of infinitely dilute solutions, it is sufficient to consider the behavior of isolated chains, where interactions (often of short range) within the chain are relevant. The case of a single linear multiblock copolymer chain 47,51 is very interesting even when only two different types (A, B) of blocks of the same length are considered (see Ref. [51] for discussion). Interestingly, multiblock copolymer chains have also close relation to the various toy-models (i.e., the HP model 52) which try to mimic the behavior of various biomacromolecules, which are formed by periodically repeated structural units ("monomers") along their chain, in order to understand complicated biological phenomena, i.e., protein folding [53, helical structures [54], etc.

Multiblock copolymers composed of two different types of blocks (A and B) under good solvent conditions form coil structures and the chain conformations are essentially dictated by the repulsive interactions between the different monomers [47,48. In fact, the chemical difference of monomers should be kept responsible for an expansion in the chain dimensions with respect to the equivalent homopolymer chains (same total number of monomers) under the same thermodynamic conditions [48 50. Also, it is expected that the spherical symmetry of the macromolecule should be distorted adopting a slightly ellipsoidal overall formation [4 51]. Nevertheless, the most interesting behavior is expected when the solvent quality is varied. Under poor solvent conditions the chain collapses, and different scenarios of microphase separation between the monomers $\mathrm{A}$ and $\mathrm{B}$ belonging to different blocks are conceivable depending on the block length and the number of blocks of the chain at a given temperature [47. Identification of these different states could be based on the analysis of the formed clusters as monomers of the same, or different, blocks join together. For rather short chains (accessible to simulations), simulation techniques provide the best way to the understanding of the phase behavior of a single multiblock copolymer, while a theoretical treatment could be an elaborate task. To the best of our 


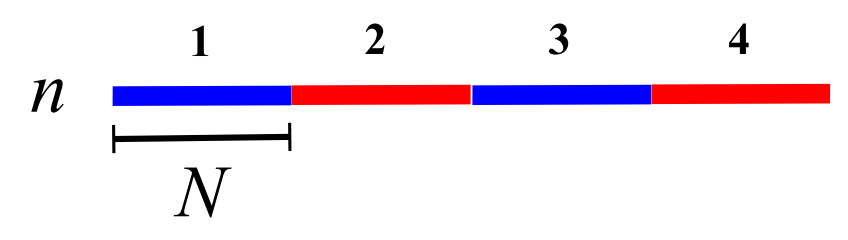

Fig. 1: (Color online) Definition of structural parameters describing our linear multiblock copolymer chains. $n$ (in this case $n=4$ ) is the number of different blocks A and B denoted with different color (or grey tone) and $N$ is the length of each block. All the blocks, irrespective of whether they are of type A or B, have the same length $N$. Then the total length of the chains is $n N$.

knowledge, the phase behavior of a single linear multiblock copolymer has not yet been discussed, even for the most symmetrical case.

In the following, we fill this gap and describe large-scale molecular dynamics (MD) simulations of an off-lattice bead-spring model of a single linear multiblock copolymer under poor solvent conditions to provide a picture for the phase behavior of such macromolecules. We remind the reader that one expects that for such a simulated system sharp phase boundaries between different states do not exist; rather smooth crossovers are expected.

In this study, we discuss results for the phase behavior of a single symmetric multiblock copolymer with regular succession of two different types of blocks (A and B) composing the linear macromolecule. By using the term "symmetric" we mean that the length of all blocks (A or B) was always equal $\left(N_{A}=N_{B}=N\right)$ and the total number of $\mathrm{A}\left(n_{A}\right)$ and $\mathrm{B}\left(n_{B}\right)$ blocks was $n\left(n=n_{A}+n_{B}\right)$, with $n_{A}=n_{B}$ in all cases. Therefore, the fraction of monomers of type $\mathrm{A}$ and $\mathrm{B}$ was always constant and equal to $f=0.5$. A schematic picture of our parameters is given in fig. 1.

Then, our chains are modelled by the standard beadspring model [55]58, where all beads interact with a truncated and shifted Lennard-Jones (LJ) potential

$$
U_{L J}^{\alpha \beta}(r)=4 \varepsilon_{L J}^{\alpha \beta}\left[\left(\sigma_{L J}^{\alpha \beta} / r\right)^{12}-\left(\sigma_{L J}^{\alpha \beta} / r\right)^{6}\right]+C, \quad r \leq r_{c}
$$

where $\alpha, \beta=A, B$ denote the different type of monomers, and the constant $C$ is defined such that the potential is continuous at the cut-off $\left(r_{c}=2.5\right)$ 60]. For simplicity, $\sigma_{L J}^{\alpha \beta}=1, k_{B}=1$, but $\varepsilon_{L J}^{A A}=\varepsilon_{L J}^{B B}=2 \varepsilon_{L J}^{A B}=1$, in order to create an unmixing tendency between monomers $\mathrm{A}$ and $\mathrm{B}$ belonging to different blocks as done in previous studies [59 61]. Therefore, $\Delta \varepsilon=\varepsilon_{L J}^{A B}-1 / 2\left(\varepsilon_{L J}^{A A}+\varepsilon_{L J}^{B B}\right)$ was kept the same throughout our simulations. The connectivity along the chain is maintained by the "finite extensible non-linear elastic" (FENE) potential

$$
U_{\mathrm{FENE}}=-\frac{1}{2} k r_{0}^{2} \ln \left[1-\left(r / r_{0}\right)^{2}\right], \quad 0<r \leq r_{0} .
$$

$U_{F E N E}\left(r \geq r_{0}\right)=\infty$, and the standard choices $r_{0}=1.5$, $k=30$ were used 55,62 .

For this model, the $\Theta$ temperature is known only rather roughly, namely $\Theta=3.0$ 62]. Being interested in $T \leq \Theta$,

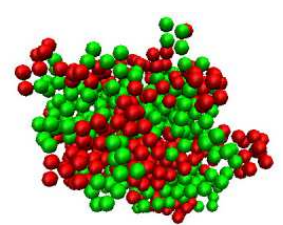

(a)

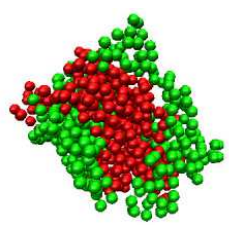

(b)

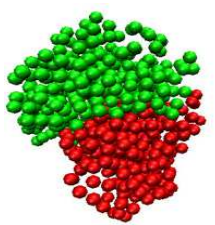

(c)
Fig. 2: (Color online) Snapshot pictures of three different multiblock copolymers of the same total length $n N=600$ at a low temperature $T=1.5$ ((a): $N=6,(\mathrm{~b}): N=15$, and (c): $N=60)$. In case (c) we have the formation for two clusters of different blocks which are always phase separated. In case (a), full phase separation does not take place and the number of clusters $N_{c l}$ has a symmetric variation around an average value $2<N_{c l}<n$. The case (b) is the intermediate case, where full microphase separation occurs with a probability $P\left(N_{c l}\right)>0$ $\left(N_{c l}=2 \neq n\right)$. Different colors (or grey tone) correspond to different type of monomers $(\mathrm{A}, \mathrm{B})$.

we simulate temperatures ranging from 1.5 to 3.0 , where the chain collapses, and monomers of A and B blocks cluster together with monomers of the same type and microphase separation between A and B monomers takes place. Using standard MD simulations [5561, the temperature was controlled by the Langevin thermostat, i.e., the equation of motion

$$
m_{L J} \frac{d^{2} \vec{r}_{i}}{d t^{2}}=-\nabla U_{i}-m_{L J} \gamma \frac{d \vec{r}_{i}}{d t}+\vec{\Gamma}_{i}(t),
$$

for the time dependence of the monomer coordinates $\vec{r}_{i}(t)$ is numerically solved. Here $t$ is the time, $U_{i}$ is the total potential the i-th bead experiences and the friction coefficient $\gamma$ was chosen $\gamma=0.5\left(m_{L J}\right.$ is also taken as unity for simplicity). The white noise $\vec{\Gamma}_{i}(t)$ satisfies the standard fluctuation dissipation relation, $\left\langle\vec{\Gamma}_{i}(t) \cdot \vec{\Gamma}_{j}\left(t^{\prime}\right)\right\rangle=$ $6 k_{B} T \gamma \delta_{i j} \delta\left(t-t^{\prime}\right)$. To equilibrate the systems close to the $\Theta$ temperature, simulations of typically $30 \times 10^{6} \tau$ were carried out at $T=3.0$, using a leap frog algorithm [63] with an integration time step of $\Delta t=0.006 \tau$, where $\tau$ is the natural time unit. After equilibration, we collect a number of independent samples (typically 500), which we use as initial configurations for slow cooling runs. For longer chains, temperatures higher than $T=3.0$ were used in order to facilitate the procedure of obtaining initial independent samples. We note here that the solvent is taken into account in our model only implicitly by tuning the temperature as done in previous work [5561. Then, decrease of the temperature corresponds to higher incompatibility of the implicit solvent with the monomers. For each cooling history, we lower the temperature in steps of $\Delta T=0.1$ and simulate the system at each temperature for a period that exceeds the relaxation time of the chains, using the final configuration at each (higher) temperature as starting configuration for the next (lower) temperature. At low enough temperatures (typically $T<2.2$ ), where dense "clusters" of a few neighboring blocks are formed, it 


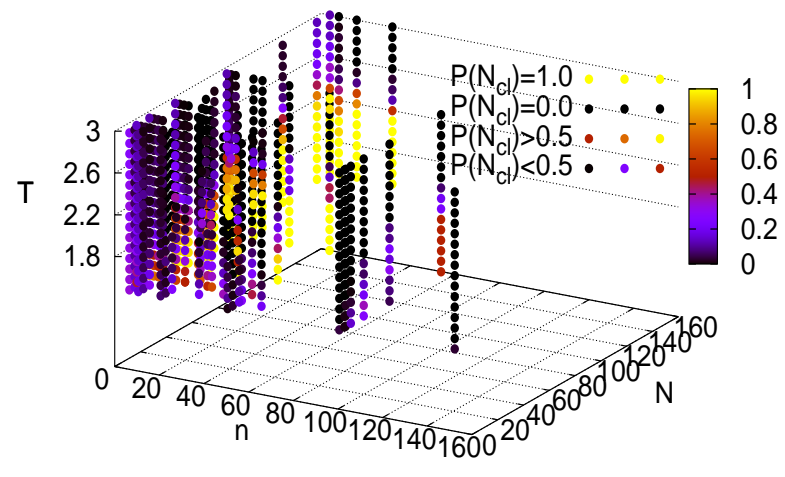

Fig. 3: (Color online) The probability of formation of a single cluster of monomers A and a single cluster of monomers B in a linear symmetric multiblock copolymer $\left(P\left(N_{c l}\right), N_{c l}=2 \neq n\right)$ is plotted for different values of block length $N$, number of blocks $n$ and temperature $T$. The different shading of colors corresponds to different values of this probability.

is not possible to run simple MD simulations long enough to sample the phase space adequately. Therefore, using this procedure of independent cooling histories is indispensable for obtaining meaningful statistical results. This large statistical effort prevents the study of exceedingly long chains, where also the relaxation time of the chains becomes very high. We have also performed runs where the temperature was changed in one step from $T=3.0$ to the desired (lower) temperature, and after equilibration, properties were calculated. While for $T \geq 2.0$ the resulting properties did not show any significant dependence on the different "history of sample preparation", somewhat more pronounced correlations are seen for temperatures lower than $T=2.0$ for a single run, which is an indication that the intrinsic relaxation times begin to exceed the available simulation time scale. Therefore, the use of independent cooling histories was necessary in order to perform a meaningful statistical analysis down to temperatures $T=1.5$.

When the chain collapses at the low temperatures and microphase separated clusters of different blocks are formed, in order to define the different types of phase behavior one needs to identify for each configuration that is analyzed, which blocks belong to a cluster. As in previous studies 64, we have used the standard Stillinger neighborhood criterion for monomers 65. When two monomers are less than a distance $r_{n}$ apart, they belong to the same cluster, but monomers $\mathrm{A}$ and $\mathrm{B}$ are always considered to belong to different clusters. We followed the standard choice $r_{n}=1.5$, but we have also checked smaller values for $r_{n}$ (i.e., $r_{n}=1.2$ ) and similar qualitative results were obtained. Higher values of $r_{n}$ are physically hardly significant, since then the monomers are too weakly bound, due to the rapid fall-off of the LJ potential. fig. 2 shows the different types of microphase separation that can be ob-

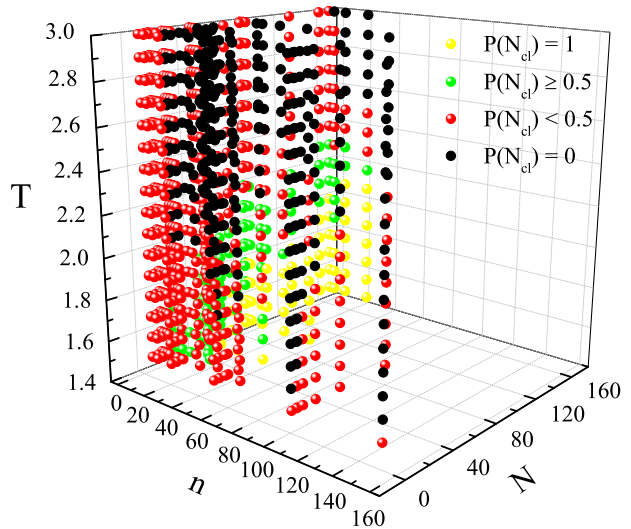

Fig. 4: (Color online) Same as in fig. 3. but the colors are considered only according to the four cases for the probability $P\left(N_{c l}\right),\left(N_{c l}=2 \neq n\right)$ as indicated for clarity.

served in the case of symmetric multiblock copolymers. In case (a), blocks of the same type can join together forming clusters, but never all the blocks of the same type are able to form a single cluster phase separated from another cluster of B monomers. In this case, the number of formed clusters $N_{c l}$ fluctuates symmetrically around an average value $\left(2<N_{c l}<n\right)$. This behavior is also an indication that the adopted simulation protocol that we followed in this work leads to a meaningful statistical analysis by considering this large number of independent cooling histories, as is done in previous work 56, 59,61, 64. Moreover, analyzing separately the properties for clusters of A monomers and for clusters of B monomers we obtain the same results, which also shows the validity of our simulation procedure. In case (c), all monomers A of all different blocks belong to a single cluster of A-type monomers and another cluster of B-type monomers is also formed. Then case (b) is the intermediate case, where strong fluctuations in the number of clusters is observed and the occurrence of full phase separation takes place with a probability $P\left(N_{c l}\right)>0.0, N_{c l}=2$. Thus, the different phase behavior in multiblock copolymers can be characterized by defining the boundaries between cases (a), (b), and (c) in terms of the probability $P\left(N_{c l}\right)$ for $N_{c l}=2$.

figs. 3 and 4 present results of the probability of detecting full phase separation (i.e., all blocks of the A-type monomers form a single cluster, while all monomers of Btype belong to another cluster $\left(P\left(N_{c l}\right), N_{c l}=2\right)$ between monomers A and monomers B. The shading of the colors in fig. 3 corresponds to this probability, whereas in fig. 4 only the colors of the four different cases are shown for clarity, without color shading according to the probability $P\left(N_{c l}=2\right)$, as indicated in the figures. Considering this probability for the full phase separation, we are able to describe the different cases of the phase behavior, which fig. 2 illustrates with characteristic snapshot pictures. We 
have considered a broad variation of parameters, $n, N$, and $T$, with $T \leq \Theta$, and we show here representative results of our simulations for the description of the different regimes. We can observe that for small block length $N$ $(N<20)$, full phase separation $\left(P\left(N_{c l}=2\right)=1\right.$, as in case (a) of fig. 2) can be hardly seen independently of the number of blocks $n$, even at low temperatures, where the chains collapse and form globules. In this regime, the probability of full phase separation is $P\left(N_{c l}=2\right)<0.5$. Looking more carefully in fig. 3 we can actually observe that decrease of $n$ increases the probability of the formation of single clusters (one with A monomers and another with B monomers), as expected.

The most interesting regime, where full microphase separation occurs, is for rather small values of $n, 4 \leq n \leq 20$ (of course for $n=2$ the clusters of A and B monomers are considered always separated). For this to happen, also the length of the blocks $N$ has to be higher than a certain value $(N \geq 20)$. This regime is indicated in figs. 3 and fig. 4 with yellow color (or the very light grey tone). It turns out that the increase of $N$ for certain value of $n$ favors full phase separation of A and B blocks, since for higher $N$ full separation is observed, even at higher temperatures. In particular, for $n=4,8,12$ and $N=150$ such an effect takes place even at temperatures in the range $T=2.3$ to $T=2.4$. At high temperatures, independently of $n$ and $N$, the probability $P\left(N_{c l}\right) \approx 0\left(N_{c l}=2\right)$. In this case, the different blocks prefer to be apart at high temperatures and can form clusters with other blocks with monomers of the same type only occasionally, due to thermal fluctuations. To conclude, full phase separation is favored when the number of blocks is low, and the block length higher than a certain value $(N \approx 20)$, for the range of temperatures that we used in our simulations. We note that the different states occur gradually and the boundaries between the different states are to be considered as smooth crossovers (indicated also by the results of fig. 33). However, we have observed with closer inspection of our data that these crossovers become sharper with the increase of the block length $N$.

Very interesting is also the phase behavior observed for high number of blocks $n$, while $N$ remains finite. In this case, $n$ is so high that the occurrence of full phase separation, as we have defined it already in our discussion, is rather not observed for the given set of potential parameters $(\Delta \varepsilon)$. What actually one sees is domains of different (A and B) microphase separated clusters for the range of $N$ presented in this study. It would be very interesting to simulate very long block lengths, in order to check if full microphase separation is possible for very high $N$ (or changing $\chi \sim \Delta \varepsilon / T$; changing either $\chi$ or $N$ would result in tremendous difficulties in simulating such a system) keeping also high the number of blocks $n$. However, if one naively tries to extrapolate our data for $n$ and $N$ in the range where full microphase separation occurs, it could be suggested that full phase separation for high $n$ could require also very high values of $N$. It has also been discussed for copolymer melts [4, that the geometry of the microphase separated regions is controlled by the number of blocks $n$, as well as other parameters, i.e., relative size and arrangement of the blocks. Nevertheless, one would expect in the long chain limit in equilibrium that a ground-state type structure would be a single lamellar domain, where an interface between all A-type and B-type blocks is formed, similarly to what is known for multiblock copolymer melts [33]. Such a structure would have much less (unfavorable) A-B contacts rather than a multidomain structure of A and B clusters, which is kinetically favored due to the preparation protocol in the simulation and the chosen set of parameters accessible to simulations.

In summary, we have discussed the microphase separation of symmetric linear multiblock copolymers, where a chain contains blocks of monomers of types A and B, which alternate along the chain via MD simulations of an off-lattice bead spring model. The fraction of monomers A $\left(f_{A}\right)$ and monomers B $\left(f_{B}\right)$ was always constant at $f=f_{A}=f_{B}=0.5$. Varying the number of blocks $n$, the block length $N$, and the temperature $T$, we were able to detect the regimes where full phase separation (all blocks of type A belong to a single cluster and all blocks of type B to another cluster), as well the regime that full phase separation does not take place. We found that rather small values of $n(4 \leq n \leq 20)$ and increasing block length $N(N>20)$ favors full separation of the blocks, but $N$ should in no case be lower than the aforementioned value. Let us point out here that, the behavior of multiblock copolymers can be parallelized with that of various biological macromolecules, which are formed by periodically repeated structural units ("monomers") along the chain. In such systems the capability of the formation of clusters could be related to the probability of forming bonds and occurrence of interactions between different blocks on the same chain. Further investigation is needed for very high number of blocks and very high block lengths, not accessible to our simulations.

\section{REFERENCES}

[1] Benoit H., J. Polym. Sci., 11 (1953) 597.

[2] Flory P. J. and ABe A., Macromolecules, 6 (1978) 1119.

[3] Glatter O. and Kratky O., Small angle X-Ray Scattering (Academic Press, New York) 1982.

[4] Fried H. and Binder K., J. Chem. Phys., 94 (1991) 8349.

[5] De Gennes P.-G., Scaling Concepts in Polymer Physics (Cornell University Press, Ithaca, London) 1988.

[6] Leibler L., Macromolecules, 13 (1980) 1602.

[7] Benoit H. and Hadziononnou G., Macromolecules, 21 (1988) 1449.

[8] Mayes A. M. and De la Cruz M., J. Chem. Phys., 91 (1989) 7228.

[9] Löwenhaupt M. and Hellmann G. P., Polymer, 32 (1991) 1065.

[10] Matsen M. and Schick M., Phys. Rev. Lett., 72 (1994) 2660. 
[11] Matsen M. W. and Schick M., Macromolecules, 27 (1994) 6761.

[12] Matsen M. W., J. Chem. Phys., 102 (1995) 3884.

[13] Fredrickson G. H. and Sides S. W., Macromolecules, 36 (2003) 5415.

[14] Fredrickson G. H. and Sides S. W., Macromolecules, 36 (2003) 5415.

[15] Erukhimovich I. Ya. and Dobrynin A. V., Macromolecules, 25 (1992) 4411.

[16] Angerman H., ten Brinke G. and Erukhimovich I., Macromolecules, 29 (1996) 3255.

[17] Dobrynin A. V. and Leibler L., Macromolecules, 30 (1997) 4756.

[18] Slog J. J. M., Angerman H. J. and ten Brinke G., J. Chem. Phys., 108 (1998) 8677.

[19] Friedel P., John A., Netz R. R., Pospiech D. and Jehnichen D., Macromol. Theory. Simul., 11 (2002) 785.

[20] John A., Friedel P., Pospiech D., Jehnichen D. and Kunert C., Macromol. Theory. Simul., 13 (2004) 702.

[21] Leibler L. and Benoit H., Polymer, 22 (1981) 195.

[22] Fredrickson G. H., Macromolecules, 24 (1991) 3456.

[23] Fredrickson G. H., Milner S. T. and Leibler L., Macromolecules, 25 (1992) 6341.

[24] Spontak R. and Zelinksi J., Macromolecules, 25 (1992) 6341.

[25] Gross C. and Paul W., Soft Matter, 6 (2010) 3273.

[26] Khandpur A. K., Förster S., Bates F. S., Hamley I. W., Ryan A. J., Bras W., Almdal K. and Mortensen K., Macromolecules, 28 (1995) 8796.

[27] Sugiyama K., Toshiyuki O., El-Magd A. A. and HiRAO A., Macromolecules, 43 (2010) 1403.

[28] Matyjaszewski K. and Xia J. H., Chem. Rev., 101 (2001) 2921.

[29] Balazs A. C., Gempe M. and Brady J. E., J. Chem. Phys., 92 (1990) 2036.

[30] Fredrickson G. H. and Milner S. T., Phys. Rev. Lett., 67 (1991) 835.

[31] Glotzer S. C., Bansil R., Gallagher P. D., Gyure M. F., Sciortino F. and Stanley H. E., Physica A, 201 (1993) 482.

[32] Leibler L., Macromolecules, 15 (1982) 1283.

[33] Matsen M. W. and Schick M., Macromolecules, 27 (1994) 7157.

[34] Halperin A. and Zhulina E. B., Europhys. Lett., 16 (1991) 337.

[35] He X., Herz J. and Guenet J.-M., Macromolecules, 20 (1987) 2003.

[36] He X., Herz J. and Guenet J.-M., Macromolecules, 21 (1988) 1757.

[37] Houdayer J. and Müller M., Macromolecules, 37 (2004) 4283.

[38] De Gennes P.-G., Faraday Discuss. Chem. Soc., 68 (1979) 96.

[39] Flory P. J., Principles of Polymer Chemistry (Cornell Univ. Press, Ithaca, NY) 1953.

[40] Fredrickson G. H. and Helfand E., J. Chem. Phys., 87 (1987) 697.

[41] Fredrickson G. H. and Binder K., J. Chem. Phys., 91 (1989) 7265.

[42] Almdal K., Rosedale J. H., Bates F. S., Wignall G. V. and Fredrickson G. H., Phys. Rev. Lett., 65 (1990) 1112 .
[43] Bates F. S. and Fredrickson G. H., Ann. Rev. Phys. Chem., 41 (1990) 525.

[44] Leibler L., Makromol. Chem., Rapid Commun., 2 (1980) 393.

[45] De Gennes P.-G., J. Phys. (Paris), 31 (1970) 235.

[46] Semenov A. N., Sov. Phys. JETP, 61 (1985) 733.

[47] Theodorakis P. E. and Fytas N. G., Soft matter, 7 (2011) 1038.

[48] Olaj O. F., Neubauer B. and Zifferer G., Macromol. Theory Simul., 7 (1998) 171.

[49] Molina L. A. and Freire J. J., Macromolecules, 28 (1995) 2705.

[50] Molina L. A., Rodriquez A. L. and Freire J. J., Macromolecules, 27 (1994) 1160.

[51] Pham T. T., Dünweg B. and Prakash J. R., Macromolecules, 43 (2010) 10084.

[52] Yue K. and Dill K. A., Proc. Natl. Acad. Sci. U. S. A., 92 (1995) 146.

[53] Creigton T. E., Protein Folding (Freeman, New York) 1994.

[54] Magee J. E., Vasquez V. R. and Lue L., Phys. Rev. Lett., 96 (2006) 207802.

[55] Grest G. S. and Kremer K., Phys. Rev. A: At., Mol., Opt. Phys., 33 (1986) 3628.

[56] Theodorakis P. E., Paul W. and Binder K., Europhys. Lett., 88 (2009) 63002.

[57] Grest G. S. and Murat M., Monte Carlo and Molecular Dynamics Simulations in Polymer Science, edited by K. Binder (Oxford University Press, New York) 1995.

[58] Grest G. S., Adv. Polym. Sci., 138 (1999) 149.

[59] Theodorakis P. E., Paul W. and Binder K., Macromolecules, 43 (2010) 5137.

[60] Das S. K., Horbach J. and Binder K., J. Chem. Phys., 119 (2003) 1547.

[61] Erukhimovich I., Theodorakis P. E., Paul W. and Binder K., J. Chem. Phys., 134 (2011) .

[62] Grest G. S. and Murat M., Macromolecules, 26 (1993) 3108.

[63] van Gunsteren W. F. and Berendsen H. J. C., Mol. Simul., 1 (1988) 173.

[64] Theodorakis P. E., Paul W. and Binder K., J. Chem. Phys., 133 (2010) 104901.

[65] Stillinger F. H., J. Chem. Phys., 38 (1963) 1486. 